\title{
Mass spectrometry quantification of clusterin in the human brain
}

Junjun Chen ${ }^{1,2}$, Meiyao Wang ${ }^{1,2}$ and Illarion V Turko ${ }^{1,2^{*}}$

\begin{abstract}
Background: The multifunctional glycoprotein clusterin has been associated with late-onset Alzheimer's disease (AD). Further investigation to define the role of clusterin in AD phenotypes would be aided by the development of techniques to quantify level, potential post-translational modifications, and isoforms of clusterin. We have developed a quantitative technique based on multiple reaction monitoring (MRM) mass spectrometry to measure clusterin in human postmortem brain tissues.

Results: A stable isotope-labeled concatenated peptide (QconCAT) bearing selected peptides from clusterin was expressed with an in vitro translation system and purified. This clusterin QconCAT was validated for use as an internal standard for clusterin quantification using MRM mass spectrometry. Measurements were performed on the human postmortem frontal and temporal cortex from control and severe AD cases. During brain tissues processing, $1 \%$ SDS was used in the homogenization buffer to preserve potential post-translational modifications of clusterin. However, MRM quantifications in the brain did not suggest phosphorylation of $\mathrm{Thr}^{393}$, $\mathrm{Ser}^{394}$, and $\mathrm{Ser}^{396}$ residues reported for clusterin in serum. MRM quantifications in the frontal cortex demonstrated significantly higher $(P<0.01)$ level of clusterin in severe AD group $(39.1 \pm 9.1 \mathrm{pmol} / \mathrm{mg}$ tissue protein) in comparison to control group $(25.4 \pm 4.4 \mathrm{pmol} / \mathrm{mg}$ tissue protein). In the temporal cortex, the clusterin levels were not significantly different, $29.0 \pm 7.9 \mathrm{pmol} / \mathrm{mg}$ tissue protein and $28.0 \pm 8.4 \mathrm{pmol} / \mathrm{mg}$ tissue protein in control and severe AD groups, respectively.
\end{abstract}

Conclusions: The proposed protocol is a universal quantitative technique to assess expression level of clusterin. It is expected that application of this protocol to quantification of various clusterin isoforms and potential post-translational modifications will be helpful in addressing the role of clusterin in AD.

Keywords: Clusterin, QconCAT, Multiple reaction monitoring, Human brain, Alzheimer's disease

\section{Background}

Genome-wide associated studies have linked the CLU gene with the risk of developing late-onset Alzheimer's disease (AD) $[1,2]$. CLU encodes clusterin, a multifunctional glycoprotein, whose involvement in $\mathrm{AD}$ has been discussed since increased clusterin expression in $\mathrm{AD}$ hippocampus was first reported two decades ago [3]. Subsequent Western blot and ELISA quantifications pointed to increased levels of clusterin in AD hippocampus and in frontal cortex [4], cerebrospinal fluid [5], and plasma [6]. However, other Western blot studies

\footnotetext{
* Correspondence: iturko@umd.edu

${ }^{1}$ Institute for Bioscience and Biotechnology Research, University of Maryland, Rockville, Maryland 20850, USA

${ }^{2}$ Analytical Chemistry Division, National Institute of Standards and

Technology, Gaithersburg, Maryland 20899, USA
}

demonstrated that levels of clusterin did not differ significantly between control and $\mathrm{AD}$ cases in the frontal cortex, temporal cortex, or thalamus in postmortem human brain $[7,8]$. The explanation for these differences is unclear, but might originate from the variety of clusterin antibodies used and availability of clusterin epitopes in the different biological samples examined. The challenge of understanding the role of clusterin in $\mathrm{AD}$ goes beyond the quantification of protein levels. Clusterin has alternative splicing variants, single nucleotide polymorphisms, and post-translational modifications $[9,10]$, which are difficult to ascertain with immunological methods and likely associated with development of $\mathrm{AD}$. Overall, the availability of a reliable quantitative approach is needed to facilitate clarification of the role of clusterin in $\mathrm{AD}$.

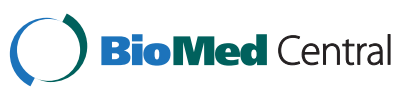

(c) 2012 Chen et al.; licensee BioMed Central Ltd. This is an Open Access article distributed under the terms of the Creative Commons Attribution License (http://creativecommons.org/licenses/by/2.0), which permits unrestricted use, distribution, and reproduction in any medium, provided the original work is properly cited. 
The application of multiple reaction monitoring (MRM) mass spectrometry to proteomics has greatly enhanced the selectivity and accuracy of protein quantification. Absolute quantification using MRM assay relies on isotope-labeled internal standards, which can be peptides [11], concatenated peptides (QconCAT) [12], or full-length proteins [13]. However, utilization of any of these internal standards has limitations. Isotope-labeled peptides provide the most high-throughput analysis, although have the highest chance of serious quantitative discrepancies between analyte and standard. Isotopelabeled full-length proteins are perfectly quantitative as internal standards, but do not support high-throughput analysis due to poor availability. QconCATs have a strong potential to be perfectly quantitative and support high-throughput analysis at the same time [14,15].

QconCATs are similar to analyte proteins, but not identical. Potentially, this can result in incomplete proteolysis of either the QconCATs or the analyte proteins and therefore affect quantification. There are two main determinants of the rate of proteolysis. First is the composition of amino acid residues around the cleavage site. The second is the tight folding of some proteins that makes them intrinsically resistant to proteolysis. We addressed these issues by (i) incorporating into the QconCATs the original flanking sequences from the proteins that surround the tryptic fragments (Q-peptides) to create an identical amino acid composition around the cleavage site, and (ii) initial treatment of biological samples with a SDS-containing buffer to completely unfold proteins. SDS in the homogenization buffer has the added advantage of preserving post-translational modifications that may be present in target proteins. The final optimized protocol was used to assess the levels of clusterin and levels of potential clusterin phosphorylation in the specimens of human brain from control and severe AD donors. The data obtained expand our understanding of the role(s) clusterin may play in AD.

\section{Results and discussion}

\section{Flanking regions for Q-peptides}

Often, the Q-peptides in the QconCAT are directly adjacent to each other. Thus, the flanking regions of Qpeptides in the standard and analyte are different and proteolysis may occur at a different rate for the QconCAT and analyte. To avoid potential quantitative discrepancy, the incorporation of native flanking sequences into the QconCATs was first proposed by Kito et al. [16] and further used by Nanavati et al. [14]. At the same time, evaluation of QconCATs without flanking sequences demonstrated that the digestion protocol had a significant impact on final quantification [17]. Figure 1A illustrates the design of our QconCAT for clusterin with five Q-peptides carrying 6-amino acid long extensions from their natural sequences on both sides of the Q-peptides. This QconCAT was expressed as an isotope-labeled protein and purified using a $\mathrm{C}$ terminal $\mathrm{His}_{6}$-tag (Figure $1 \mathrm{~B}$ ). The $\mathrm{His}_{6}$-tag is separated from the last Q-peptide by the flanking sequence specific for that Q-peptide and therefore will not affect the rate of proteolytic release. Additionally, the presence of flanking sequences eliminates concerns about dibasic cleavage sites and acidic residues at P2' position and allows for greater flexibility in QconCAT design.

Optimal MRM transitions for Q-peptides were experimentally selected from analysis of clusterin QconCAT tryptic digestion. These transitions were then used to determine the level of stable isotope incorporation in the clusterin QconCAT. Signals from $\mathrm{Q}_{2}$ - and $\mathrm{Q}_{3}$-peptides were low and these two peptides were not used for further quantifications. Figure $1 \mathrm{C}$ shows representative extracted ion chromatograms for $\mathrm{Q}_{1}, \mathrm{Q}_{4}$, and $\mathrm{Q}_{5}$ peptides. It is evident that both light and heavy versions of the peptides are present. This is presumably due to contaminating unlabeled Arg or Lys amino acids, or synthesis, in the in vitro kit. The isotope incorporation factor, $\alpha$, is calculated as the ratio of the area of the labeled peak to the sum of the unlabeled and labeled peaks. The two strongest transitions per peptide were used to calculate isotope incorporation for each peptide. Combined data for these three peptides resulted in a final value of $0.75 \pm 0.02$. A single point calibration was used to assign a concentration value based on the following formula:

$$
\begin{aligned}
\operatorname{clusterin}(\mathrm{pmol} / \mathrm{mg}) & =\left[\left(\frac{\mathrm{A}}{\mathrm{S}}+1\right) \mathrm{x} \alpha-1\right] \\
& \mathrm{x} \frac{\text { standard }(\mathrm{pmol})}{\text { tissue protein }(\mathrm{mg})}
\end{aligned}
$$

where $\mathrm{A}$ is the analyte peak area, $\mathrm{S}$ is the standard peak area, and $\alpha$ is the isotope incorporation factor. The equation functions to shift the light component of the $Q$ peptide from the analyte peak area to the standard peak area proportionally based on the isotope incorporation factor.

The MRM transitions used for all following quantifications are summarized in Table 1.

\section{SDS-containing buffer for sample processing}

QconCATs are artificial proteins, which are not expected to be folded into higher order structures. Nevertheless, on the level of QconCAT design, we selected only those peptides from clusterin which are not involved in $\beta$ sheet formation in the native clusterin to avoid a potential resistance to proteolysis. To ensure full unfolding of the native clusterin and equal proteolytic rates for analyte (native clusterin) and internal standard (clusterin QconCAT), $25 \mathrm{mM} \mathrm{NH}_{4} \mathrm{HCO}_{3}$ containing $1 \%(\mathrm{~m} / \mathrm{V})$ 


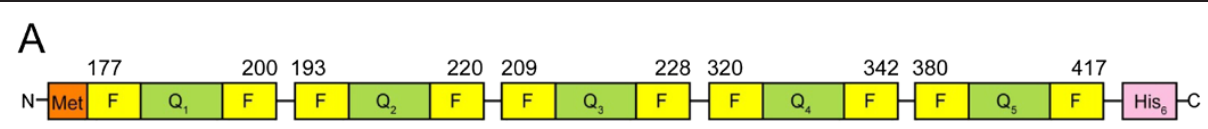

B

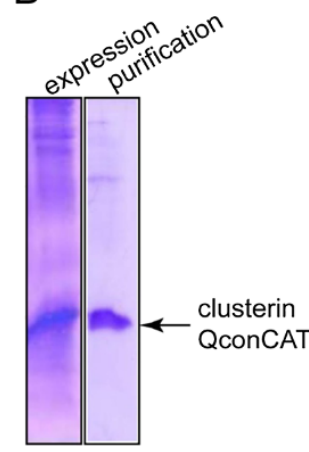

C

isotope incorporation factor: $0.75 \pm 0.02$
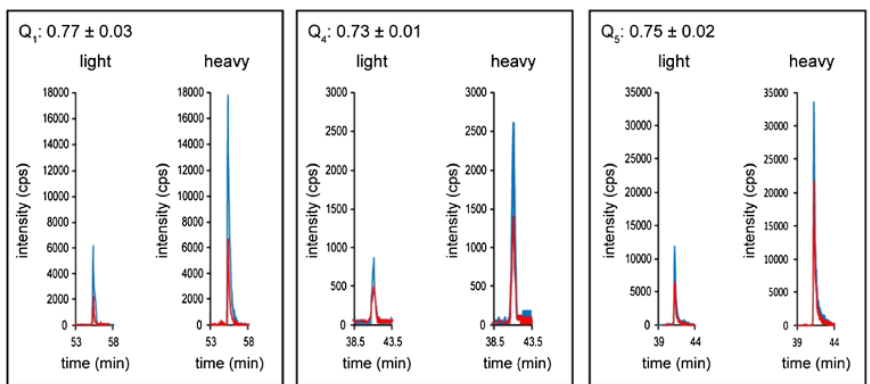

Figure 1 Design, expression, and characterization of clusterin QconCAT. (A). Clusterin QconCAT includes five Q peptides (shown in green) with 6-amino acid long flanking regions (shown in yellow) concatenated into the sequence with N-terminal Met and C-terminal His ${ }_{6}$-tag. (B) Coomassie R250 stained clusterin QconCAT after 15\% polyacrylamide gel separation. (C) Stable isotope incorporation into the clusterin. MRM spectra for two representative transitions per $\mathrm{Q}_{1}, \mathrm{Q}_{4}$, and $\mathrm{Q}_{5}$ peptides are shown. The pair transitions for light (unlabeled) and heavy (labeled) form of each Q peptide are color coordinated. Isotope incorporation factor for clusterin QconCAT was calculated based on combined data for all three peptides and presented as mean \pm SD.

SDS was selected as a homogenization buffer for tissue processing. In addition, SDS-containing buffer for biological sample processing immediately terminates any enzymatic reactions that can change the levels of present post-translational modifications. After supplementation with QconCAT and alkylation of Cys residues, samples were precipitated with chloroform/methanol to remove SDS, biological lipids, and by-products of alkylation. The resulting pellet of pure and fully unfolded proteins was further proteolysed and analyzed by MRM.

\section{Validation of proteolytic digestion}

To determine the completeness of digestion, we performed trypsin digestions of human recombinant clusterin (purity $>95 \%$, ProSpec-Tany Technogene, Ness Ziona, Israel) and clusterin QconCAT mixed in different ratios. Table 2 demonstrates that, based on three peptides and two different ratios, the average difference between mixed concentrations and actually measured is approximately $16 \%$. In addition to the rate of proteolysis, this combined error also includes the errors generated by mixing of clusterins and the MRM assay itself. Overall, this difference is very acceptable for quantification and the data confirm that proteolytic digestion of our clusterin QconCAT accurately reproduces proteolytic digestion of native full-length clusterin.

\section{Quantification of clusterin}

The methodological improvements described above demonstrated that flanking sequences for Q-peptides in QconCAT in combination with full-unfolding of native proteins in 1\% SDS prevent potential inconsistency in the tryptic digestion and reduce the bias in quantification. To finalize the protocol for clusterin quantification in brain tissues, the dynamic range and limit of quantification (LOQ) were determined in temporal cortex homogenates. The standard curves were generated for $\mathrm{Q}_{1^{-}}, \mathrm{Q}_{4^{-}}$, and $\mathrm{Q}_{5^{-}}$ peptides by mixing different concentrations of labeled

Table 1 Peptides and MRM transitions used for the quantification of clusterin

\begin{tabular}{|c|c|c|c|c|}
\hline \multirow{3}{*}{$\begin{array}{l}\text { Peptides } \\
\text { ASSIIDELFQDR }\left(Q_{1}, \text { light }\right)\end{array}$} & \multicolumn{4}{|c|}{ MRM transitions $(\mathrm{m} / \mathrm{z})$} \\
\hline & \multirow{2}{*}{$\begin{array}{l}\text { Precursor } \\
697.35(+2)\end{array}$} & \multicolumn{3}{|c|}{ Product } \\
\hline & & $678.40\left(y^{5},+1\right)$ & $922.43\left(y^{7},+1\right)$ & $1035.51\left(y^{8},+1\right)$ \\
\hline ASSIIDELFQDR ( $Q_{1}$, heavy) & $702.36(+2)$ & $688.40\left(y^{5},+1\right)$ & $932.43\left(y^{7},+1\right)$ & $1045.52\left(y^{8},+1\right)$ \\
\hline ELDESLQVAER ( $Q_{4}$, light) & $644.82(+2)$ & $602.30\left(y^{5},+1\right)$ & $802.44\left(y^{7},+1\right)$ & $1046.51\left(y^{9},+1\right)$ \\
\hline ELDESLQVAER ( $Q_{4}$, heavy) & $649.83(+2)$ & $612.30\left(y^{5},+1\right)$ & $812.45\left(y^{7},+1\right)$ & $1056.52\left(y^{9},+1\right)$ \\
\hline VTTVASHTSDSDVPSGVTEWVK ( $\mathrm{Q}_{5}$, light) & $772.06(+3)$ & $507.80\left(y^{10},+2\right)$ & $773.48\left(y^{7},+1\right)$ & $1014.58\left(y^{10},+1\right)$ \\
\hline VTTVASHTSDSDVPSGVTEWVK ( $\mathrm{Q}_{5}$, heavy) & $774.74(+3)$ & $511.80\left(y^{10},+2\right)$ & $781.49\left(y^{7},+1\right)$ & $1022.60\left(y^{10},+1\right)$ \\
\hline
\end{tabular}

$\left[{ }^{13} \mathrm{C}_{6}{ }^{15} \mathrm{~N}_{2}\right]$-Lys and $\left[{ }^{13} \mathrm{C}_{6},{ }^{15} \mathrm{~N}_{4}\right]$-Arg in labeled peptides are shown in bold. For MRM transitions, charge for precursor ions, and, and the type and charge for product ions are shown in parentheses. 
Table 2 Validation of proteolytic digestion

\begin{tabular}{lll}
\hline & \multicolumn{2}{l}{$\begin{array}{l}\text { Recombinant clusterin/QconCAT } \\
\text { clusterin (pmol/pmol) }\end{array}$} \\
\cline { 2 - 3 } & Mixed & Measured \\
\hline ASSIIDELFQDR $\left(\mathrm{Q}_{1}\right)$ & 1.0 & $1.2 \pm 0.1$ \\
ELDESLQVAER $\left(\mathrm{Q}_{4}\right)$ & 2.0 & $2.4 \pm 0.2$ \\
& 1.0 & $1.3 \pm 0.2$ \\
VTTVASHTSDSDVPSGVTEWVK $\left(\mathrm{Q}_{5}\right)$ & 1.0 & $1.9 \pm 0.3$ \\
& 2.0 & $1.2 \pm 0.2$ \\
consensus & 2.0 & $2.4 \pm 0.3$ \\
\hline
\end{tabular}

The measurements were performed for three experimental replicates by monitoring three transitions per individual peptide and presented as mean $\pm \mathrm{SD}$. For consensus, the mean measured ratio is presented as a percentile of mean mixed ratio.

clusterin QconCAT (0.1 - 10 pmoles range) into the $0.2 \mathrm{mg}$ of temporal cortex homogenate. The data were plotted for every individual transition and showed linearity and low scatter. Figure 2 shows a representative standard curve for each $\mathrm{Q}$ peptide. LOQ was defined as the lowest standard point of the curve that could be measured with a coefficient of variance less than $20 \%$. The LOQ was 3 $\mathrm{pmol} / \mathrm{mg}$ of tissue protein for $\mathrm{Q}_{1}$ - and $\mathrm{Q}_{5}$-peptides. The measurements based on $\mathrm{Q}_{4}$-peptide were less sensitive with LOQ equal to $10 \mathrm{pmol} / \mathrm{mg}$ of tissue protein. For this reason, $\mathrm{Q}_{4}$-peptide was not used for the following quantifications in the human brain.

Clusterin was quantified (summarized in Table 3) in human frontal and temporal cortex brain samples obtained from two groups of donors, control with no or minimal $\mathrm{AD}$ changes (clinical dementia rating 0) and severe $\mathrm{AD}$ cases (clinical dementia rating 3) [for demographic information of the donors, see Additional file 1]. Notably, the measurements based on the $\mathrm{Q}_{1}$-peptide (ASSIIDELFQDR) and those based on $\mathrm{Q}_{5}$-peptide (VTTVASHTSDSDVPSGVTEVVVK) are similar. Phosphorylation of $\mathrm{Thr}^{393}$, Ser ${ }^{394}$, or $\mathrm{Ser}^{396}$ in VTTVASHTSDSDVPSGVTEVVVK has been reported for clusterin present in serum [10]. Our protocol for sample processing includes tissue homogenization in the presence of $1 \%$ SDS which should prevent loss of post-translational modifications. If any modifications were present in $Q_{5}$, the measurements based on $Q_{5}$ would be lower (than based on $\mathrm{Q}_{1}$ ) proportionally to the level of modification. However, we did not observe a statistically significant difference between quantifications based on $\mathrm{Q}_{1}$ and $\mathrm{Q}_{5}$. This may point to a very low level of $\mathrm{Thr}^{393}, \mathrm{Ser}^{394}$, or $\mathrm{Ser}^{396}$ phosphorylation in the brain, if any.

The second important observation is that the level of clusterin in frontal cortex is significantly higher $(P<0.01)$ in severe $\mathrm{AD}(39.1 \pm 9.1 \mathrm{pmol} / \mathrm{mg}$ tissue protein) in comparison to control $(25.4 \pm 4.4 \mathrm{pmol} / \mathrm{mg}$ tissue protein) while the clusterin levels in the temporal cortex are not significantly different, $29.0 \pm 7.9 \mathrm{pmol} / \mathrm{mg}$ tissue protein and $28.0 \pm 8.4 \mathrm{pmol} / \mathrm{mg}$ tissue protein in control and severe $\mathrm{AD}$ groups, respectively. These quantifications point to a selective increase of clusterin level in the specific region of AD brain.

\section{Conclusions}

We have developed an antibody-free protocol for clusterin quantification in tissue samples. The protocol includes tissue homogenization in 1\% SDS that will be helpful in discovery and validation of clusterin isoforms and post-translational modifications. The subsequent chloroform/methanol precipitation is a simple approach to remove SDS before trypsinolysis and MRM. In addition, we have used isotope-labeled QconCAT with original flanking regions for every Q-peptide and demonstrated that this internal standard entirely matched to full-length clusterin in the MRM quantitative assay. Subsequent measurements of clusterin in frontal and temporal cortex from control and severe AD donors showed that the proposed protocol can be used to address the role of clusterin in AD.

\section{Methods}

Materials

Expressway cell-free E.coli expression system was from Invitrogen (Carlsbad, CA). L- $\left[{ }^{13} \mathrm{C}_{6},{ }^{15} \mathrm{~N}_{2}\right]$ lysine and
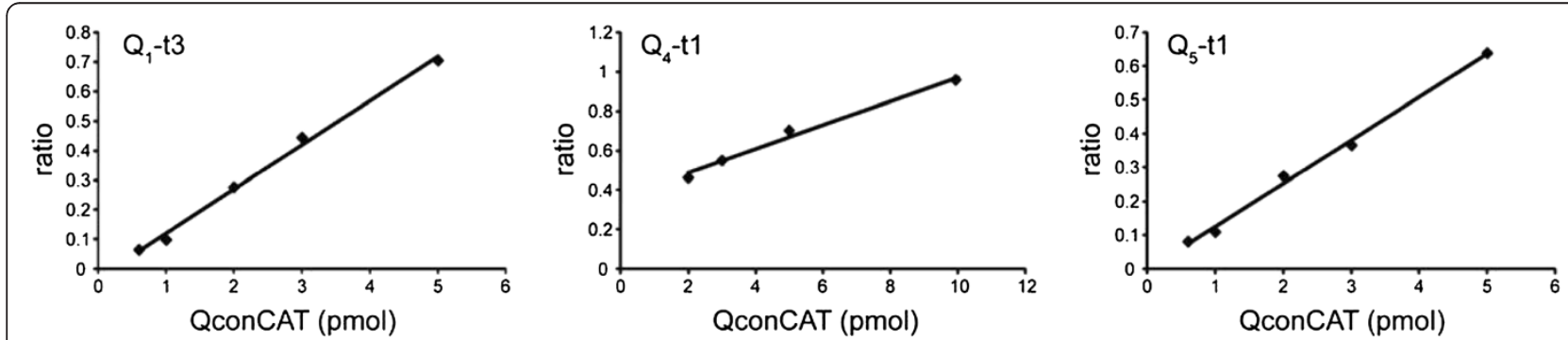

Figure 2 Representative standard curves for $\mathbf{Q}_{1}, \mathbf{Q}_{4}$, and $\mathbf{Q}_{5}$ peptides. The area ratio of heavy to light peaks for a selected transition was plotted versus suplemented clusterin QconCAT amount for each Q peptide. Transitions: $\mathrm{Q}_{1}$-t3 is 697.35/1035.51 and 702.36/1045.52; $\mathrm{Q}_{4}$-t1 is 644.82/602.30 and 649.83/612.30; $\mathrm{Q}_{5}$-t1 is 772.06/507.80 and 774.74/511.80. 
Table 3 Quantification of clusterin in the human brain tissues

\begin{tabular}{lllll}
\hline & \multicolumn{2}{c}{ Clusterin, pmol/mg tissue protein } \\
\cline { 2 - 5 } & \multicolumn{2}{c}{ Frontal cortex } & \multicolumn{2}{c}{ Temporal cortex } \\
\cline { 2 - 5 } & Control & Severe AD & Control & $29.5 \pm 7.4$ \\
ASSIIDELFQDR $\left(Q_{1}\right)$ & $26.0 \pm 4.2$ & $39.6 \pm 9.0$ & $2.0 \pm 8.5$ \\
VITVASHTSDSDVPSGVTEWVK $\left(Q_{5}\right)$ & $24.8 \pm 4.7$ & $38.6 \pm 9.3$ & $27.8 \pm 7.9$ & $29.3 \pm 7.7$ \\
consensus & $25.4 \pm 4.4$ & $39.1 \pm 9.1$ & $29.0 \pm 7.9$ & $28.0 \pm 8.4$ \\
\hline
\end{tabular}

Each control and severe AD group includes six human donors. The concentration was calculated for three experimental replicates by monitoring three transitions per individual peptide and presented as mean \pm SD. The monitored transitions are summarized in Table 1. The reported phosphorylated Thr and Ser residues [10] are underlined. For the consensus, the data for both peptides were combined and presented as mean \pm SD. Clusterin concentrations in frontal cortex were significantly different $(P<0.01)$ using a $t$ test comparison for the control and severe AD groups, whereas clusterin concentrations in temporal cortex were not significantly different.

L- $\left[{ }^{13} \mathrm{C}_{6},{ }^{15} \mathrm{~N}_{4}\right]$ arginine (>95\% purity) were from Spectral Stable Isotopes (Columbia, MD). The DC Protein Assay kit was from Bio-Rad Laboratories (Hercules, CA). Sequencing grade modified trypsin was obtained from Promega Corp. (Madison, WI). Recombinant human clusterin (purity $>95 \%$ ) was from ProSpec-Tany Technogene, Ness Ziona, Israel. All other chemicals were purchased from Sigma-Aldrich (St. Louis, MO).

\section{Design, expression and purification of clusterin QconCAT} A synthetic gene encoding 131 amino acids composed of the sequence MQDHFSRASSIIDELFQDRFFTREPDRFFTREPQDTYHYLPFSLPHRRPHFFFFSLPHRRPHFFFPKSRIVRSQAKLRRELDESLQVAERLTRKYNDQYYLRVTTVASHTSDSDVPSGVTEVVVKLFDSDP was synthesized by Integrated DNA Technologies (Coralville, Iowa). The underlined segments represent the signature proteotypic peptides of clusterin (Q-peptides). The synthetic clusterin gene was cloned into the $\mathrm{pEXP5CT/TOPO} \mathrm{expres-}$ sion vector in-frame to the $\mathrm{C}$-terminal $\mathrm{His}_{6}$-tag. Stable isotope-labeled clusterin QconCAT was expressed using an in vitro translation kit (Invitrogen) according to the manufacture's protocol. L- $\left[{ }^{13} \mathrm{C}_{6},{ }^{15} \mathrm{~N}_{2}\right]$ lysine and L$\left[{ }^{13} \mathrm{C}_{6},{ }^{15} \mathrm{~N}_{4}\right]$ arginine were added to the amino acid mixture to replace unlabeled lysine and arginine. After in vitro translation, the labeled clusterin QconCAT was purified by nickel-nitrilotriacetic acid resin in batch mode (Qiagen, Valencia, CA). Finally the purified QconCAT was loaded onto the SpinTrap G-25 spin column (GE Healthcare, Waukesha, WI) to exchange buffer into $25 \mathrm{mM} \mathrm{NH}_{4} \mathrm{HCO}_{3}$ with $1 \%(\mathrm{~m} / \mathrm{V})$ SDS. The protein concentration of clusterin QconCAT was measured in the presence of $1 \%(\mathrm{~m} / \mathrm{V})$ SDS using the $D C$ protein Assay kit and bovine serum albumin as a standard. The final clusterin QconCAT was aliquoted and kept frozen at $-80^{\circ} \mathrm{C}$.

\section{Human tissues}

Samples of frontal cortex were received from the Washington University School of Medicine Alzheimer's Disease Research Center. Samples of temporal cortex were obtained from the Alzheimer's Disease Center, Boston University. All brain specimens were collected from deidentified donors following informed consent of the respective families.

\section{Processing of samples}

The minced brain tissue was placed in $25 \mathrm{mM}$ $\mathrm{NH}_{4} \mathrm{HCO}_{3} / 1 \%(\mathrm{~m} / \mathrm{V})$ SDS and homogenized by sonication at $30 \mathrm{~W}$ using five $10 \mathrm{~s}$ continuous cycles (Sonicator 3000, Misonix Inc., Farmingdale, NY). The homogenate was centrifuged at $2000 \mathrm{~g}$ for $5 \mathrm{~min}$ to remove tissue debris. The supernatant was used to measure total protein concentration in the presence of $1 \%$ SDS using the $D C$ protein Assay kit and bovine serum albumin as a standard. The supernatant was then aliquoted $0.2 \mathrm{mg}$ of total tissue protein per tube and kept frozen at $-80^{\circ} \mathrm{C}$. During the following experiments, samples of $0.2 \mathrm{mg}$ of total tissue protein were supplemented with $20 \mathrm{mM}$ DTT and various amounts of labeled clusterin QconCAT, ranging from $0.1 \mathrm{pmol}$ to $10 \mathrm{pmol}$ per sample. The mixture was incubated at room temperature for 60 min to allow reduction of cysteines and was then treated with $50 \mathrm{mM}$ iodoacetamide for another $60 \mathrm{~min}$. Alkylated samples were precipitated with chloroform/ methanol [18]. The protein pellets obtained were sonicated in $100 \mu \mathrm{L}$ of $25 \mathrm{mM} \mathrm{NH}_{4} \mathrm{HCO}_{3}$ and treated with trypsin for $15 \mathrm{~h}$ at $37^{\circ} \mathrm{C}$. The substrate/trypsin ratio was 50:1 $(\mathrm{m} / \mathrm{m})$. After trypsinolysis, the samples were dried using a vacuum centrifuge (Vacufuge, Eppendorf AG, Hamburg, Germany).

\section{LC-MS/MS analysis}

Instrumental analyses were performed on a hybrid triple quadrupole/linear ion trap mass spectrometer (4000 QTRAP, ABI/MDS-Sciex) coupled to an Eksigent nanoLC-2D system (Dublin, CA). Separation of peptides was performed with an Eksigent cHiPLC- nanoflex system equipped with a nano cHiPLC column, $15 \mathrm{~cm} \mathrm{x}$ $75 \mu \mathrm{m}$, packed with ReproSil-Pur C18-AQ, $3 \mu \mathrm{m}$ (Dr. Maisch, Germany). Peptides were eluted over a 29 min-gradient from $15 \%$ to $35 \%$ acetonitrile, 
containing $0.1 \%$ formic acid at a flow rate of $300 \mathrm{~nL} /$ $\mathrm{min}$. The column effluent was continuously directed into the nanospray source of the mass spectrometer. All acquisition methods used the following parameters: an ion spray voltage of $2200 \mathrm{~V}$, curtain gas of $105 \mathrm{kPa}$ (15 psi), source gas of $140 \mathrm{kPa}(20 \mathrm{psi})$, interface heating temperature of $170^{\circ} \mathrm{C}$, declustering potential of $76 \mathrm{~V}$ for +2 precursor ions and $65 \mathrm{~V}$ for +3 precursor ions, collision energy of $30 \mathrm{~V}$ for +2 precursor ions and $22 \mathrm{~V}$ for +3 precursor ions, and collision cell exit potential of $16 \mathrm{~V}$ for +2 precursor ions and $13 \mathrm{~V}$ for +3 precursor ions. The dwell time for all transitions was $40 \mathrm{~ms}$.

\section{Quantitative analysis and validation}

The initial list of MRM transitions was selected as described [19] and was experimentally screened for the three most intensive transitions per peptide. These transitions were further used for quantification. The relative ratios of the three transitions monitored in the $25 \mathrm{mM}$ $\mathrm{NH}_{4} \mathrm{HCO}_{3}$ for labeled clusterin QconCAT were similar to those observed by spiking labeled clusterin QconCAT into the biological samples. This indicated no significant interference for the quantification based on selected transitions. The identities of the measured peptides were confirmed based on the retention time of the three MRM peaks from a given peptide and the ratio among the three MRM peaks. The reported value for each group was the mean and SD of each biological replicate using all peptide transitions.

\section{Additional file}

Additional file 1: Donors of temporal and frontal cortex.

\section{Competing interests}

The authors declare no competing financial interest.

\section{Authors' contributions}

$J C, M W$, and IVT conceived and designed the experiments. JC expressed and characterized the isotope labeled clusterin QconCAT. IVT performed MS sample preparation. JC and MW performed MS data acquisition and analysis. IVT wrote the paper. All authors revised the manuscript and gave final approval of the version to be published.

\footnotetext{
Acknowledgements

This work was supported in part by the Washington University School of Medicine Alzheimer's Disease Research Center grant (P50 AG05681). Certain commercial materials, instruments, and equipment are identified in this manuscript in order to specify the experimental procedure as completely as possible. In no case does such identification imply a recommendation or endorsement by the National Institute of Standards and Technology nor does it imply that the materials, instruments, or equipment identified are necessarily the best available for the purpose.
}

Received: 8 June 2012 Accepted: 14 August 2012

Published: 20 August 2012

\section{References}

1. Harold D, Abraham R, Hollingworth P, Sims R, Gerrish A, Hamshere ML, Pahwa JS, Moskvina V, Dowzell K, Williams A, et al: Genome-wide association study identifies variants at CLU and PICALM associated with Alzheimer's disease. Nat Genet 2009, 41:1088-1093.

2. Lambert JC, Heath S, Even G, Campion D, Sleegers K, Hiltunen M, Combarros O, Zelenika D, Bullido MJ, Tavernier B, et al: Genome-wide association study identifies variants at CLU and CR1 associated with Alzheimer's disease. Nat Genet 2009, 41:1094-1099.

3. May PC, Lampert-Etchells M, Johnson SA, Poirier J, Masters JN, Finch CE: Dynamics of gene expression for a hippocampal glycoprotein elevated in Alzheimer's disease and in response to experimental lesions in rat. Neuron 1990, 5:831-839.

4. Lidstrom AM, Bogdanovic N, Hesse C, Volkman I, Davidsson P, Blennow K: Clusterin (apolipoprotein J) protein levels are increased in hippocampus and in frontal cortex in Alzheimer's disease. Exp Neurol 1998, 154:511-521.

5. Nilselid AM, Davidsson P, Nagga K, Andreasen N, Fredman P, Blennow K: Clusterin in cerebrospinal fluid: analysis of carbohydrates and quantification of native and glycosylated forms. Neurochem Int 2006 48:718-728.

6. Thambisetty M, Simmons A, Velayudhan L, Hye A, Campbell J, Zhang Y, Wahlund LO, Westman E, Kinsey A, Guntert A, et al: Association of plasma clusterin concentration with severity, pathology, and progression in Alzheimer disease. Arch Gen Psychiatry 2010, 67:739-748.

7. Harr SD, Uint L, Hollister R, Hyman BT, Mendez AJ: Brain expression of apolipoproteins E, J, and A-I in Alzheimer's disease. J Neurochem 1996, 66:2429-2435

8. Baig S, Palmer LE, Owen MJ, Williams J, Kehoe PG, Love S: Clusterin mRNA and protein in Alzheimer's disease. J Alzheimers Dis 2012, 28:337-344.

9. Leskov KS, Klokov DY, Li J, Kinsella TJ, Boothman DA: Synthesis and functional analyses of nuclear clusterin, a cell death protein. J Biol Chem 2003, 278:11590-11600.

10. Zhou W, Ross MM, Tessitore A, Ornstein D, Vanmeter A, Liotta LA, Petricoin EF 3rd: An initial characterization of the serum phosphoproteome. J Proteome Res 2009, 8:5523-5531.

11. Gerber SA, Rush J, Stemman O, Kirschner MW, Gygi SP: Absolute quantification of proteins and phosphoproteins from cell lysates by tandem MS. Proc Natl Acad Sci U S A 2003, 100:6940-6945.

12. Beynon RJ, Doherty MK, Pratt JM, Gaskell SJ: Multiplexed absolute quantification in proteomics using artificial QCAT proteins of concatenated signature peptides. Nat Methods 2005, 2:587-589.

13. Brun V, Dupuis A, Adrait A, Marcellin M, Thomas D, Court M, Vandenesch F, Garin J: Isotope-labeled protein standards: toward absolute quantitative proteomics. Mol Cell Proteomics 2007, 6:2139-2149.

14. Nanavati D, Gucek M, Milne JL, Subramaniam S, Markey SP: Stoichiometry and absolute quantification of proteins with mass spectrometry using fluorescent and isotope-labeled concatenated peptide standards. Mol Cell Proteomics 2008, 7:442-447.

15. Bislev SL, Kusebauch U, Codrea MC, Beynon RJ, Harman VM, Rontved CM, Aebersold R, Moritz RL, Bendixen E: Quantotypic properties of QconCAT peptides targeting bovine host response to Streptococcus uberis. J Proteome Res 2012, 11:1832-1843.

16. Kito K, Ota K, Fujita T, Ito T: A synthetic protein approach toward accurate mass spectrometric quantification of component stoichiometry of multiprotein complexes. J Proteome Res 2007, 6:792-800.

17. Mirzaei $\mathrm{H}, \mathrm{McBee} J \mathrm{~K}$, Watts J, Aebersold R: Comparative evaluation of current peptide production platforms used in absolute quantification in proteomics. Mol Cell Proteomics 2008, 7:813-823.

18. Liao WL, Turko IV: Strategy combining separation of isotope-labeled unfolded proteins and matrix-assisted laser desorption/ionization mass spectrometry analysis enables quantification of a wide range of serum proteins. Anal Biochem 2008, 377:55-61

19. Liao WL, Heo GY, Dodder NG, Pikuleva IA, Turko IV: Optimizing the conditions of a multiple reaction monitoring assay for membrane proteins: quantification of cytochrome P450 11A1 and adrenodoxin reductase in bovine adrenal cortex and retina. Anal Chem 2010, 82:5760-5767.

doi:10.1186/1750-1326-7-41

Cite this article as: Chen et al: Mass spectrometry quantification of clusterin in the human brain. Molecular Neurodegeneration 2012 7:41. 